Для цитирования: Максимова И.В. Когнитивные и электроэнцефалографические изменения у пациентов с алкогольной зависимостью, перенесших судорожный припадок. Сибирский вестник психиатрии и наркологии. $2018 ; 2$ (99): 89-92. https://doi.org/10.26617/1810-3111-2018-2(99)-89-92

\title{
Когнитивные и электроэнцефалографические изменения у пациентов с алкогольной зависимостью, перенесших судорожный припадок
}

\section{Максимова И.В.}

Красноярский государственный медицинский университет имени проф. В. Ф. Войно-Ясенечкого Россия, 660022, Красноярск, ул. Партизана Железняка, д. 1

\section{PEЗЮME}

Введение. Прогрессирующее нарушение интеллектуальных функций является характерной особенностью алкогольной зависимости. Когнитивные нарушения у лиц, страдающих хроническим алкоголизмом, выявляются в 50-70\% случаев, в 10\% случаев они носят выраженный характер, достигающий степени деменции. Материалы и методы. Обследовано 149 пациентов с алкогольной зависимостью II-III стадии. Когнитивные нарушения оценивались с помощью Краткой шкалы оценки психического статуса (MMSE), также проводилось электроэнцефалографическое исследование. Результаты. Электроэнцефалографическое обследование у пациентов, перенесших генерализованный тонико-клонический приступ, отличалось отсутствием характерных эпи-феноменов. Также при проведении ЭЭГ было выявлено снижение мощности основного ритма и увеличение удельного веса медленно-волновой активности тета- и дельта-диапазонов. Сравнительный анализ когнитивных нарушений по шкале MMSE в результате лечения показал различия в восстановлении когнитивных функций между группами. Заключение. Проведение адекватной терапии препаратами, стимулирующими нейротрансмиттерные системы (пептиды, холинергики, нейрометаболиты, блокаторы NMDA-рецепторов), витаминами и нормализация питания приводят к восстановлению когнитивных функций.

Ключевые слова: алкогольная зависимость, алкогольные психозы, большой судорожный припадок, когнитивные расстройства.

\section{ВВЕДЕНИЕ}

Современные методы дезинтоксикации привели к снижению летальности при экзогенных интоксикациях, связанных с алкоголем [1]. В то же время отмечается увеличение количества больных, прежде всего работоспособного возраста, с сопутствующим органическим поражением ЦНС на фоне сформированной алкогольной зависимости $[2,3]$.

Эпилептический синдром является одним из проявлений токсического действия этанола на головной мозг. Многочисленные литературные данные свидетельствуют о коморбидности зависимости от алкоголя и других, в том числе психических и неврологических заболеваний $[4,5,6]$. В настоящее время не выявлены специфические ЭЭГ-признаки эпилептиформных пароксизмов, позволяющие уточнить нозологическую принадлежность этих расстройств и их отличительные признаки. Между тем раннее выявление больных наиболее объективными методами представляет значительную актуальность в плане их дальнейшего наблюдения [7].

Цель исследования - оценить изменения когнитивных функций и результатов электроэнцефалографического исследования в ходе лечения у пациентов, страдающих алкогольной зависимостью и перенесших генерализованный тонико-клониеский приступ.

\section{МАТЕРИАЛЫ И МЕТОДЫ}

Bce участники исследования находились на момент оценки их состояния на стационарном лечении в Красноярском краевом наркологическом диспан- сере № 1. Диагноз алкогольной зависимости (F10.*) устанавливался в рамках МКБ-10. Основную группу составили 83 пациента, перенесшие генерализованный тонико-клонический приступ (ГТКП) на фоне зависимости от алкоголя и обратившиеся за медицинской помощью в связи с развитием абстиненции или делирия. Группа сравнения включала пациентов с алкогольной зависимостью без ГТКП на момент поступления и составила 66 человек.

Обследование больных осуществлялось клинико-психопатологическим методом, принятым в современной психиатрии. Для оценки когнитивных нарушений использовалась Краткая Шкала Оценки Психического Статуса (MMSE), статистические методы. Статистическая обработка полученных данных выполнялась при помощи программы SPSS, версии 22.0 .

Описательная статистика результатов исследования представлена для качественных и порядковых признаков в виде процентных долей и их стандартных ошибок, для количественных - в виде средних арифметических $(\mathrm{M})$ и стандартных отклонений $(\sigma)$. Значения средних арифметических имеют вид $\mathrm{M} \pm \sigma$. Различия во всех случаях оценивали как статистически значимые при $\mathrm{p}<0,05$.

\section{РЕЗУЛЬТАТЫ И ОБСУЖДЕНИЕ}

Всем пациентам, участвующим в исследовании, было проведено обследование и лечение согласно стандартам оказания наркологической помощи. Сроки госпитализации больных без генерализованного тонико-клонического приступа составили 12 
дней, с ГТКП - 14 дней. Среди обследованных пациентов наличие ГТКП отмечалось у 83 лиц $(55,7 \%)$. В большинстве случаев $(63-76,0 \%)$ приступы возникали в остром периоде алкогольного абстинентного синдрома и развивались в течение 48 часов от последнего употребления алкоголя или при снижении дозы.

Средний возраст больных основной группы составил $40,7 \pm 11,9$ года, что оказалось существенно ниже среднего возраста пациентов группы сравнения $(43,1 \pm 12,8$ года). В основной группе давность заболевания более чем у трети пациентов составила более 15 лет (37,3\%), давность заболевания в группе сравнения оказалась несколько меньшей $(28,8 \%)$, однако значимых различий между группами обследованных не выявлено (табл. 1).

Т а б л и ц а 1

Давность заболевания пациентов

\begin{tabular}{|l|c|c|}
\hline $\begin{array}{c}\text { Давность } \\
\text { заболевания }\end{array}$ & $\begin{array}{c}\text { Основная группа } \\
(\mathrm{n}=83)\end{array}$ & $\begin{array}{c}\text { Группа сравнения } \\
(\mathrm{n}=66)\end{array}$ \\
\hline $2-5$ лет & $17(20,5 \%)$ & $21(31,8 \%)$ \\
\hline $6-10$ лет & $21(25,3 \%)$ & $13(19,7 \%)$ \\
\hline $11-15$ лет & $14(16,9 \%)$ & $13(19,7 \%)$ \\
\hline Более 15 лет & $31(37,3 \%)$ & $19(28,8 \%)$ \\
\hline
\end{tabular}

К особенностям формирования и течения алкогольной зависимости в основной группе можно отнести более длительный период злоупотребления алкоголем (12 лет - в основной группе и 10,5 года в группе сравнения) и более короткие ремиссии (2 и 4 месяца соответственно) (табл. 2).

Т а б л и ц а 2 Особенности течения алкогольной зависимости

\begin{tabular}{|l|c|c|}
\hline \multicolumn{1}{|c|}{ Показатель } & $\begin{array}{c}\text { Основная группа } \\
(\mathrm{n}=83)\end{array}$ & $\begin{array}{c}\text { Группа сравнения } \\
(\mathrm{n}=66)\end{array}$ \\
\hline $\begin{array}{l}\text { Возраст начала употребле- } \\
\text { ния алкоголя, лет }\end{array}$ & $16[14 ; 18]$ & $17[15 ; 18]$ \\
\hline Стаж злоупотребления, лет & $12 *[6 ; 18,5]$ & $10,5[5 ; 16,75]$ \\
\hline Длительность запоев, дни & $10[7 ; 14]$ & $14[7 ; 21]$ \\
\hline $\begin{array}{l}\text { Длительность ремиссий, } \\
\text { месяцы }\end{array}$ & $2 *[1 ; 6]$ & $4[1,25 ; 6]$ \\
\hline
\end{tabular}

У $32(38,6 \%)$ пациентов основной группы в анамнезе отмечено наличие черепно-мозговой травмы, в группе сравнения ЧМТ зафиксированы с меньшей частотой - у $9(13,6 \%)$ обследуемых $(\mathrm{p}<0,001)$. Это может рассматриваться как предиктор развития ГТКП при алкоголизме и подтверждает мнение о значимости сопутствующего травматического поражения головного мозга.

Возникновение приступов обычно носило внезапный характер, не отмечалось предвестников или ауры, у всех пациентов приступы протекали с полной утратой сознания. Они носили генерализованный характер, однако чаще протекали без прикуса языка. На фоне течения алкогольной зависимости структура ГТКП не усложнялась, чаще всего они носили стереотипный, единичный характер, завершались сном. Проведенное в тот же день или ближайшие дни после приступа электроэнцефалографическое обследование отличалось отсутствием характерных эпи-феноменов.
Также при проведении ЭЭГ было выявлено снижение общей амплитуды основного ритма (альфаритма): в основной группе общая амплитуда составила $50 \pm 6,7$ мкВ, в группе сравнения общая амплитуда составила $72 \pm 3,4$ мкВ, на уровне статистической тенденции прослежено увеличение удельного веса медленно-волновой активности тета- и дельтадиапазонов.

Генерализованные тонико-клонические приступы, предшествовавшие развитию делириозного состояния, возникали у 10 больных $(12,1 \%)$. У 3 (3,6\%) из них отмечалось серийное течение припадков. ЭЭГ-данные спустя 2 дня после приступов у всех трех обследуемых пациентов характеризовались диффузной высокоамплитудной дизритмией. Так называемая алкогольная эпилепсия отмечалась у 4 $(4,8 \%)$ обследуемых при длительном злоупотреблении алкоголем. Судорожные припадки, которые начались при злоупотреблении алкоголем, в последующем сохранялись вне зависимости от алкогольных эксцессов. Время возникновения судорожных приступов не зависело от состояния абстиненции или трезвости. Воздержание от употребления алкогольных напитков не устраняло судорожные приступы. На ЭЭГ, выполненной через двое суток после приступа, отмечалась битемпоральная эпилептическая активность.

После поступления пациентов в стационар ГТКП повторились у 15 пациентов $(18,1 \%)$, на третьи сутки от начала медикаментозного лечения состояние стабилизировалось и ГТКП не повторялись.

Пациентам проводилось лечение с использованием карбамазепина, препаратов, стимулирующих нейротрансмиттерные системы (пирацетам, мемантин), витаминотерапия (витамины группы В).

После лечения отмечалась положительная динамика в неврологическом статусе у пациентов группы сравнения в виде отсутствия субъективных жалоб, уменьшения неврологической недостаточности: снижение тремора головы и конечностей - у 13 (20\%) пациентов. В основной группе значимых различий в изменении неврологического статуса не наблюдалось. Субъективно обследуемыми отмечалось улучшение состояния, которое можно связать с отменой алкоголя на период лечения.

Обследование когнитивных нарушений по шкале MMSE проводилось на 3-4-й день госпитализации и перед выпиской. Сравнительный анализ полученных данных показал значимые различия между группами. До лечения в основной группе средний балл составил 24 (min-max 18-29), в группе сравнения - 27 (min-max 23-30). После комплексной терапии достоверно повышался суммарный показатель в баллах по шкале MMSE: до 29 (min-max 25-30) - в группе сравнения и до 27 (min-max 22-30) - в основной группе. Однако в подгруппе пациентов, перенесших делирий с судорожным синдромом, улучшение когнитивных функций было менее выражено, что связано с тяжестью состояния и сопутствующим органическим поражением ЦНС (табл. 3). 


\begin{tabular}{|l|c|c|c|c|}
\hline \multicolumn{5}{c|}{ Т а б л и ц а 3} \\
\hline \multirow{2}{*}{ Показатель } & \multicolumn{5}{|c|}{ Основная группа } & Группа сравнения \\
\cline { 2 - 6 } & $\begin{array}{c}\text { До лече- } \\
\text { ния }\end{array}$ & $\begin{array}{c}\text { После } \\
\text { лечения }\end{array}$ & $\begin{array}{c}\text { До лече- } \\
\text { ния }\end{array}$ & $\begin{array}{c}\text { После } \\
\text { лечения }\end{array}$ \\
\hline Средний балл ММSE & 24 & 27 & 27 & 29 \\
\hline Минимальное значение & 18 & 22 & 23 & 25 \\
\hline Максимальное значение & 29 & 30 & 30 & 30 \\
\hline
\end{tabular}

\section{ЗАКЛЮЧЕНИЕ}

Для контроля эффективности терапии больных основной группы применялось ЭЭГ-исследование. При проведении ЭЭГ у больных, перенесших состояние отмены с судорогами или алкогольный делирий с судорогами, было выявлено снижение мощности основного ритма, на уровне статистической тенденции прослежено увеличение удельного веса медленно-волновой активности тета- и дельтадиапазонов.

При назначении терапии карбамазепином анализ ЭЭГ в динамике позволял проводить коррекцию терапии: нарастание на ЭЭГ эпилептиформной активности, особенно в виде билатерально-синхронных разрядов, свидетельствовало о резистентности к карбамазепину и целесообразности замены препарата на вальпроевую кислоту. Таким образом, прекращение злоупотребления алкоголем и проведение адекватной терапии приводит к восстановлению когнитивных функций в баллах по шкале MMSE: до 29 (min-max 25-30) - в группе сравнения и до 27 (min-max 22-30) - в основной группе.

\section{КОНФЛИКТ ИНТЕРЕСОВ}

Автор заявляет об отсутствии конфликта интересов в связи с публикацией данной статьи.

\section{ИСТОЧНИК ФИНАНСИРОВАНИЯ}

Автор заявляет об отсутствии финансирования при проведении исследования.

\section{СООТВЕТСТВИЕ ПРИНЦИПАМ ЭТИКИ}

Работа соответствует этическим стандартам Хельсинской декларации ВМА (протокол заседания этического комитета ГБОУ ВПО «Красноярский государственный медицинский университет им. проф. В.Ф. Войно-Ясенецкого» Минздрава России № 59/2014 от 02 декабря 2014 г.).

\section{ЛИТЕРАТУРА}

1. Zanjani F., Downer B.G., Kruger T.M., Willis S.L., Schaie K.W Alcohol effects on cognitive change in middle-aged and older adults. Aging. Ment. Health. 2013; 17(1): 12-23.

2. Зиновьева О.Е., Емельянова А.Ю. Неврологические проявления алкогольной болезни: роль витаминов группы В в лечении. Consilium Medicum. 2016; 18 (9): 133-138.

3. WHO. Global status report on alcohol and health. Geneva: World Health Organization, 2014.

4. Андрианова Е.Д., Дамулин И.В., Сиволап Ю.П. Когнитивные расстройства при алкоголизме. Наркология. 2013; 12 (138): 79-85.

5. Катаманова Е.В., Рукавишников В.С., Лахман О.Л., Шевченко О.И., Денисова И.А. Когнитивные нарушения при токсическом поражении мозга. Журнал неврологии и психиатрии им. С.С. Корсакова. 2015; 115 (2-1): 11-15.

6. Бохан Н.А., Иванова С.А., Мандель А.И., Жернова Е.В., Кисель Н.И. Когнитивные функции и процессы апоптоза у больных алкоголизмом: эффекты нейрометаболической коррекции. Наркология. 2012; 7: 51-55.

7. Казенных Т.В. Методологические подходы к реабилитации больных пароксизмальными состояниями. Сибирский вестник психиатрии и наркологии. 2014; 2 (83): 74-77.

8. Софронов А.Г., Егоров А.Ю. Клинико-фармакологическое обоснование применения карбамазепина в комплексной терапии алкоголизма. Неврологический вестник имени В.М. Бехтерева. 2013; XLV (1): 77-86.

9. Bernardin F., Maheut-Bosser A., Paille F. Cognitive impairments in alcohol-dependent subjects. Front Psychiatry. 2014; 5: 78.

10. Campagna F., Montagnese S., Schiff S., Ruzzoli M., Biancardi A., Iannizzi P., Pujatti P.L., Angeli P., Gatta A., Merkel C., Leandro G., Mapelli D., Amodio P. Confounders in the detection of minimal hepatic encephalopathy: a neuropsychological and quantified EEG study. Liver Int. 2015; 35 (5): 1524-1532.

11. Cao R., Wu Z., Li H., Xiang J., Chen J. Disturbed connectivity of EEG functional networks in alcoholism: a graph-theoretic analysis. Biomed. Mater. Eng. 2014; 24 (6): 2927-2936.

12. de la Monte S.M., Kril J.J. Human alcohol-related neuropathology. Acta Neuropathol. 2014; 127 (1): 71-90.

13. Gray J.C., Mac Killop J. Using Behavior Economics to Understand Alcohol Use Disorders: a Concise Review and Identification of Research Priorities. Current Addiction Reports. 2015; 2 (1): 68-75.

14. Welch K.A. Alcohol consumption and brain health. BMJ. 2017; $6 ; 357$.

15. Stavro K., Pelletier J., Potvin S. Widespread and sustained cognitive deficits in alcoholism: a meta-analysis. Addict. Biol. 2013; 18 (2): 203-213.

Поступила в редакцию 30.01.2018 Утверждена к печати 2.04.2018

Максимова Ирина Викторовна, ассистент кафедры психиатрии и наркологии с курсом ПО.

Максимова Ирина Викторовна, zabigylina@mail.ru

УДК 616.89-008.441.13:616.8-009.24:616-073.97

For citation: Maksimova I.V. Cognitive and electroencephalographic changes in patients undergoing a seizure, suffering from alcohol dependence. Siberian Herald of Psychiatry and Addiction Psychiatry. 2018; 2 (99): 89-92. https://doi.org/10.26617/18103111-2018-2(99)-89-92

\section{Cognitive and electroencephalographic changes in patients undergoing a seizure, suffering from alcohol dependence}

\section{Maksimova I.V.}

Krasnoyarsk State Medical University named after Prof. V. F. Voino-Yasenetsky

Partizan Zheleznyak Street 1, 660022, Krasnoyarsk, Russian Federation 


\section{ABSTRACT}

Introduction. Progressive impairment of intellectual functions is a characteristic feature of alcohol dependence. Cognitive disorders in persons suffering from chronic alcoholism are detected in 50-70\% of cases, in $10 \%$ of cases they are pronounced, reaching the degree of dementia. Materials and methods. 149 patients with stage II-III alcohol dependence are examined. Cognitive impairment is assessed using a Short scale assessment of mental status (MMSE), EEG investigation is performed. Results. Electroencephalographic examination is characterized by absence of typical EPI phenomena. Also during EEG a decrease in the power of the main rhythm and an increase in the specific weight of slow wave activity of theta and Delta bands are revealed. A comparative analysis of cognitive impairment according to the MMSE scale as a result of treatment shows significant differences between groups. Conclusion. Appropriate therapy (vitamin therapy, nutrition normalization, use of drugs that stimulate neurotransmitter systems (peptides, cholinergic agents, neurometabolites, NMDA receptor blockers) leads to recovery of cognitive functions.

\section{Keywords: alcohol dependence, alcoholic psychosis, tonic-clonic seizure, cognitive disorder.}

\section{REFERENCES}

1. Zanjani F., Downer B.G., Kruger T.M., Willis S.L., Schaie K.W Alcohol effects on cognitive change in middle-aged and older adults. Aging. Ment. Health. 2013; 17 (1): 12-23.

2. Zinov'eva O.E., Emeljanova A.Ju. Nevrologicheskie projavlenija alkogol'noj bolezni: rol vitaminov gruppy B v lechenii [Neurological manifestations of alcoholic disease: the role of vitamins in the treatment]. Consilium Medicum. 2016; 18 (9): 133-138 (in Russian).

3. WHO. Global status report on alcohol and health. Geneva: World Health Organization, 2014.

4. Andrianova E.D., Damulin I.V., Sivolap Ju.P. Kognitivnye rasstrojstva pri alkogolizme [Cognitive disorders in alcoholism]. Narkologija - Narcology. 2013; 12 (138): 79-85 (in Russian).

5. Katamanova E.V., Rukavishnikov V.S., Lakhman O.L., Shevchenko O.I., Denisova I.A. Kognitivnye narushenija pri toksicheskom porazhenii mozga [Cognitive impairment in toxic brain damage]. Zhurnal nevrologii i psihiatrii im. S.S. Korsakova - S.S. Korsakov Journal of Neurology and Psychiatry. 2015; 115 (2-1): 11-15 (in Russian).

6. Bokhan N.A., Ivanova S.A., Mandel A.I., Zhernova E.V., Kise N.I. Kognitivnye funkcii i processy apoptoza $u$ bol'nyh alkogolizmom: jeffekty nejrometabolicheskoj korrekcii [Cognitive functions and processes of apoptosis in patients with alcoholism: effects of neurometabolic correction]. Narkologija Narcology. 2012; 7: 51-55 (in Russian)

7. Kazennykh T.V. Metodologicheskie podhody k reabilitacii bolnyh paroksizmalnymi sostojanijami [Methodological approaches to rehabilitation of patients with paroxysmal conditions]. Sibirskij vestnik psihiatrii $i$ narkologii - Siberian Herald of Psychiatry and Addiction Psychiatry. 2014; 2 (83): 74-77 (in Russian).
8. Sofronov A.G., Egorov A.Yu. Kliniko-farmakologicheskoe obosnovanie primenenija karbamazepina $\mathrm{v}$ kompleksnoj terapii alkogolizma [Clinical and pharmacological rationale for the use of carbamazepine in the treatment of alcoholism]. Nevrologicheskij vestnik im. V.M. Behtereva - V.M. Bekhterev Neurological Bulletin. 2013; XLV (1): 77-86 (in Russian).

9. Bernardin F., Maheut-Bosser A., Paille F. Cognitive impairments in alcohol-dependent subjects. Front Psychiatry. 2014; 5: 78 .

10. Campagna F., Montagnese S., Schiff S., Ruzzoli M., Biancardi A., Iannizzi P., Pujatti P.L., Angeli P., Gatta A., Merkel C., Leandro G., Mapelli D., Amodio P. Confounders in the detection of minimal hepatic encephalopathy: a neuropsychological and quantified EEG study. Liver Int. 2015; 35 (5): 1524-1532.

11. Cao R., Wu Z., Li H., Xiang J., Chen J. Disturbed connectivity of EEG functional networks in alcoholism: a graph-theoretic analysis. Biomed. Mater. Eng. 2014; 24 (6): 2927-2936.

12. de la Monte S.M., Kril J.J. Human alcohol-related neuropathology. Acta Neuropathol. 2014; 127 (1): 71-90.

13. Gray J.C., Mac Killop J. Using Behavior Economics to Understand Alcohol Use Disorders: a Concise Review and Identification of Research Priorities. Current Addiction Reports. 2015; 2 (1): 68-75.

14. Welch K.A. Alcohol consumption and brain health. BMJ. 2017; $6 ; 357$.

15. Stavro K., Pelletier J., Potvin S. Widespread and sustained cognitive deficits in alcoholism: a meta-analysis. Addict. Biol. 2013; 18 (2): 203-213.

Received January 30.2018 Accepted April 2.2018

Maksimova Irina V., assistant to the Department of Psychiatry and Narcology with a course of postgraduate education, Krasnoyarsk State Medical University named after Prof. V. F. Voino-Yasenetsky, Krasnoyarsk, Russian Federation.

Maksimova Irina V., zabigylina@mail.ru 